\title{
Transferência nervosa da raiz de C4 para C5 em lesões do plexo braquial. Estudo anatômico e descrição de técnica cirúrgica*
}

\section{Nerve root transfer from C4 to C5 in brachial plexus injuries. Anatomical study and description of the surgical technique}

\author{
Gabriel Vique Valeriano ${ }^{10}$ Yussef Ali Abdouni ${ }^{1} \quad$ Antonio Carlos Da Costa ${ }^{1}$ \\ ${ }^{1}$ Grupo de Cirurgia da Mão e Microcirurgia, Departamento de \\ Endereço para correspondência Gabriel Vique Valeriano, Rua \\ Ortopedia e Traumatologia, Santa Casa de São Paulo (DOT/ISCMSP), \\ Martinico Prado, 284, apto 53, Vila Buarque, São Paulo, SP, Brasil \\ São Paulo, SP, Brasil \\ (e-mail: gviquev@hotmail.com).
}

Rev Bras Ortop 2022;57(3):443-448

\section{Resumo \\ Palavras-chave \\ - plexo braquial \\ - plexo cervical \\ - transferência de nervo \\ - raízes nervosas espinhais}

Abstract
Objetivo Estudo anatômico das raízes usadas na transferência nervosa de C4 para C5 nas lesões altas do plexo braquial, com demonstração da técnica cirúrgica.

Métodos Dissecção de 15 plexos braquiais de cadáveres de ambos os sexos, registro e análise das características morfológicas das raízes de $\mathrm{C} 4$ e $\mathrm{C5}$ e simulação de neurotização.

Resultados As características morfológicas encontradas nas raízes de C4 e C5 em todas as dissecções permitiram a mobilização das mesmas e a realização de uma neurotização sem a necessidade de usar enxerto nervoso. A técnica cirúrgica permitiu preservar ramos nervosos importantes na região abordada.

Conclusão Com base nos dados encontrados no presente estudo, podemos concluir que é possível realizar a transferência entre C4 e C5 sem provocar déficit neurológico adicional nas lesões altas de plexo braquial.

Objective This is an anatomical study of the $\mathrm{C} 4$ and $\mathrm{C} 5$ roots for nerve transfers in upper brachial plexus injuries, with surgical technique demonstration.

Methods Fifteen brachial plexuses from both male and female cadavers were dissected. The morphological features of the C4 and C5 roots were recorded and analyzed, followed by a neurotization simulation.
Trabalho desenvolvido no Departamento de Ortopedia e Traumatologia do Hospital da Irmandade da Santa Casa da Misericórdia de São Paulo (DOT/ISCMSP), São Paulo, SP, Brasil. recebido

17 de Junho de 2020

aceito

14 de Outubro de 2020

Publicado on-line

Março 31, 2021
DOI https://doi.org/

10.1055/s-0040-1722575.

ISSN 0102-3616. (c) 2021. Sociedade Brasileira de Ortopedia e Traumatologia. All rights reserved.

This is an open access article published by Thieme under the terms of the Creative Commons Attribution-NonDerivative-NonCommercial-License, permitting copying and reproduction so long as the original work is given appropriate credit. Contents may not be used for commercial purposes, or adapted, remixed, transformed or built upon. (https://creativecommons.org/ licenses/by-nc-nd/4.0/)

Thieme Revinter Publicações Ltda., Rua do Matoso 170, Rio de Janeiro, RJ, CEP 20270-135, Brazil 
Keywords

- brachial plexus

- cervical plexus

- nerve transfer

- spinal nerve roots
Results In all dissections, the morphological features of the $\mathrm{C} 4$ and $\mathrm{C} 5$ roots allowed their mobilization and neurotization with no need for a nerve graft. The surgical technique spared important regional nerve branches.

Conclusion Based on these data, we conclude that $\mathrm{C} 4-\mathrm{C} 5$ nerve transfers are feasible and result in no additional neurological deficit in upper brachial plexus injuries.

\section{Introdução}

As lesões do plexo braquial representam de 10 a $20 \%$ das lesões do sistema nervoso periférico, e são causadas, na maioria dos casos, por traumas de alta energia. ${ }^{1}$ Acometem mais frequentemente indivíduos jovens e economicamente ativos, resultando em importante limitação, tanto nas atividades de vida diária, como nas profissionais. ${ }^{2-5}$

De acordo com o nível de lesão, são comumente classificadas em lesões superiores, que acometem as raízes de C5-C6 ou C5-C6-C7; inferiores (C8-T1); ou totais. ${ }^{6}$ Kaiser et al., ${ }^{7}$ descreveram uma prevalência de lesões completas em $53 \%$, seguidas de lesões do plexo superior, com 39\%, e lesões do plexo inferior, com $6 \%$. A gravidade destas lesões varia de neuropraxia, geralmente com resolução espontânea, à lesão completa por avulsão, sem nenhum potencial para recuperação. ${ }^{8}$

Alguns autores, como Verdins e Kapickis, ${ }^{9}$ descreveram que o comprometimento do tronco superior ( $\mathrm{C} 5-\mathrm{C} 6$ ) resulta em incapacidade significativa, com perda da função do ombro (abdução e rotação externa), flexão do cotovelo e supinação do antebraço.

As estratégias para reparação do plexo braquial consistem na exploração cirúrgica seguida de reconstrução, utilizando enxerto de nervo ou transferência nervosa. A reconstrução com enxerto encontra-se reservada apenas para as lesões pós-ganglionares. Nas lesões pré-ganglionares (aquelas nas quais ocorreu avulsão da raiz), os cotos proximais não estão disponíveis para reparação com enxerto e a abordagem cirúrgica é baseada em transferências nervosas. ${ }^{3,8,10,11}$

Nas avulsões das raízes que formam o tronco superior, as opções para transferência nervosa são escassas, incluindo os nervos acessório, frênico e intercostais. Segundo Abdouni et al. ${ }^{12}$ e Malessy et al., ${ }^{13}$ a transferência do nervo acessório para o nervo supraescapular, uma das mais realizadas na reconstrução do plexo braquial, tem algumas limitações já descritas na literatura.

Yamada et al., ${ }^{14-16}$ em 1991, descreveram a transferência das raízes de C3 e C4 para reconstrução das avulsões do tronco superior (C5 e C6) usando enxerto de nervo. Já Yang et al. ${ }^{17}$ conseguiram, em um estudo anatômico, coaptar a porção avulsionada de $\mathrm{C} 5$ à raiz de $\mathrm{C} 4$ realizando laminectomia e dissecção intracanal para conseguir acessar a região acometida. A vantagem desta transferência seria a reativação da contração dos músculos escápulo-umerais e devolver, assim, os movimentos do ombro (rotação externa e abdução) realizando uma única neurotização.

O objetivo do presente estudo é avaliar as características anatômicas das raízes do plexo braquial e cervical, demostrando se é possível realizar a transferência nervosa da raiz de C4 para a raiz de C5, sem a necessidade da utilização de enxerto de nervo.

\section{Material e Método}

O estudo foi realizado através de disseção microcirúrgica de plexos braquiais em cadáveres para identificação e caracterização dos nervos cervicais C4, C5 e C6. Foram dissecados 15 plexos de 10 cadáveres, sendo 8 plexos em cadáveres do sexo masculino e 7 em cadáveres do sexo feminino, e foram anotados os dados antropométricos, tais como sexo, raça, idade, peso e altura.

Foram excluídos aqueles cadáveres com afecção neuromuscular conhecida e outras lesões ou procedimentos prévios no local da dissecção.

Todas as dissecções foram realizadas pelo mesmo pesquisador e com auxílio de lupa cirúrgica com aumento de 3,5 vezes. Foram registrados os parâmetros anatômicos das raízes cervicais C4, C5 e C6, tais como comprimento, direção e a distância entre elas. As medições foram feitas com fita milimetrada e paquímetro digital.

\section{Dissecção}

Após colocação de um coxim interescapular, foi realizada uma via paralela à borda posterior do músculo esternocleidomastoideo no seu terço médio (ponto nervoso) de aproximadamente $8 \mathrm{~cm}$ de comprimento com extensão supraclavicular (- Figura 1). A dissecção passou pelo subcutâneo, músculo platisma e fáscia profunda. A veia jugular externa foi encontrada no subcutâneo descendo superficialmente ao músculo esternocleidomastoideo. A veia jugular posterior que drena na jugular externa também foi encontrada nesta região e foi ligada para facilitar o acesso a estruturas profundas. 0 músculo esternocleidomastoideo e a veia jugular externa foram rebatidos anteromedialmente expondo o tronco superior (formado por C5 e C6) localizado entre os músculos escalenos anterior e médio. Imediatamente superior à raiz de $\mathrm{C} 5$, foi identificada a raiz do C4 num plano mais superficial (-Figura 2a). Foram identificados, emergindo da raiz de $\mathrm{C} 5$, ramos para o nervo frênico (localizado na face anterior do $\mathrm{m}$. escaleno anterior) e o nervo dorsal da escápula. Também foi possível identificar os ramos para o nervo frênico, para os músculos escalenos, elevador da escápula, e comunicantes para o nervo acessório emergindo de $\mathrm{C} 4$.

A seguir, foram realizadas medições do comprimento e diâmetro dos nervos, além da distância que separa ambos nervos após sua saída do forâmen intervertebral.Um parâmetro 


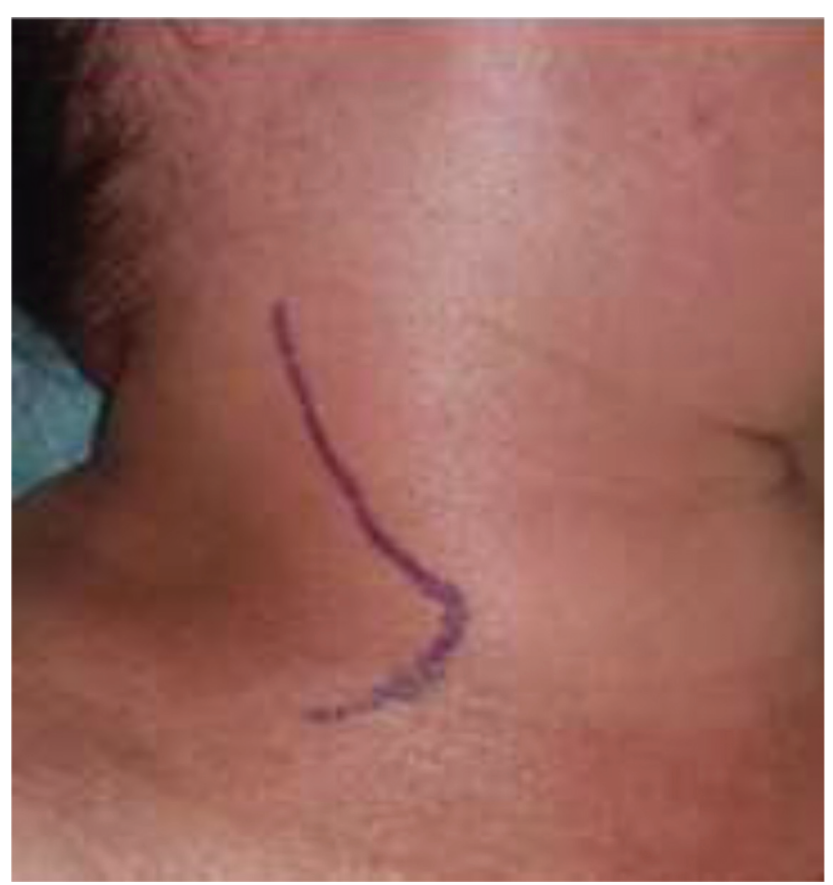

Fig. 1 Região cervical lateral direita com desenho da via usada.

importante para realizar essas medições foi a emergência do nervo frênico em C4 e C5. Finalmente, foi realizada uma simulação de transferência entre as duas raízes no cadáver (-Figura 2b), seccionando a raiz de C4 distal à emergência do nervo frênico, e tomando o cuidado de incluir nela o maior número de ramos que estariam destinados à inervação dos músculos escalenos e elevador da escápula, com o objetivo de aumentar a quantidade de fibras motoras presentes nessa raiz para neurotização. A origem do nervo frênico na raiz de $C 5$ pode ser liberada para facilitar a sua mobilização.

As medições foram feitas com o paquímetro digital Digimess - Quadrimensional $150 \mathrm{~mm}$ (Digimess Instrumentos de Precisão Ltda, São Paulo, SP, Brazil).

\section{Resultados}

$\mathrm{Na}$ - Tabela 1, mostra-se os dados antropométricos dos cadáveres dissecados. A média de idade foi de 62 anos, variando entre 38 e 86 anos. A altura máxima foi de $182 \mathrm{~cm}$ e a mínima de $152 \mathrm{~cm}$, com uma média de $167 \mathrm{~cm}$.

A - Tabela 2 mostra os resultados das medições realizadas nas raízes de $\mathrm{C} 4$ e C5. Um parâmetro importante na dissecção foi a emergência do nervo frênico nessas duas raízes como também o intervalo entre elas. Tomando em conta que a raiz de C4 encontre-se íntegra, a origem do nervo frênico foi respeitada, e só o segmento imediatamente distal a ele foi considerado útil para a mobilização e neurotização. A raiz de C5 foi medida em todo seu comprimento, tanto antes como depois da emergência do nervo frênico, e todo seu comprimento foi considerado útil para mobilização e neurotização.

Os casos 6 e 7 apresentaram a menor distância entre as raízes na região dos foramens vertebrais ( $9 \mathrm{~mm}$ ), bem como uma menor diferença no comprimento entre as raízes de C4 e C5 (12 mm), ao passo que os casos 4, 12 e 13 apresentaram uma maior distância entre as raízes de C4 e C5 (11 mm); mesmo assim, a diferença no comprimento entre as raízes sempre foi maior em todos os casos (16, 14 e $13 \mathrm{~mm}$ ). Essa diferença de comprimento entre as raízes permitiu realizar uma sutura sem tensão.
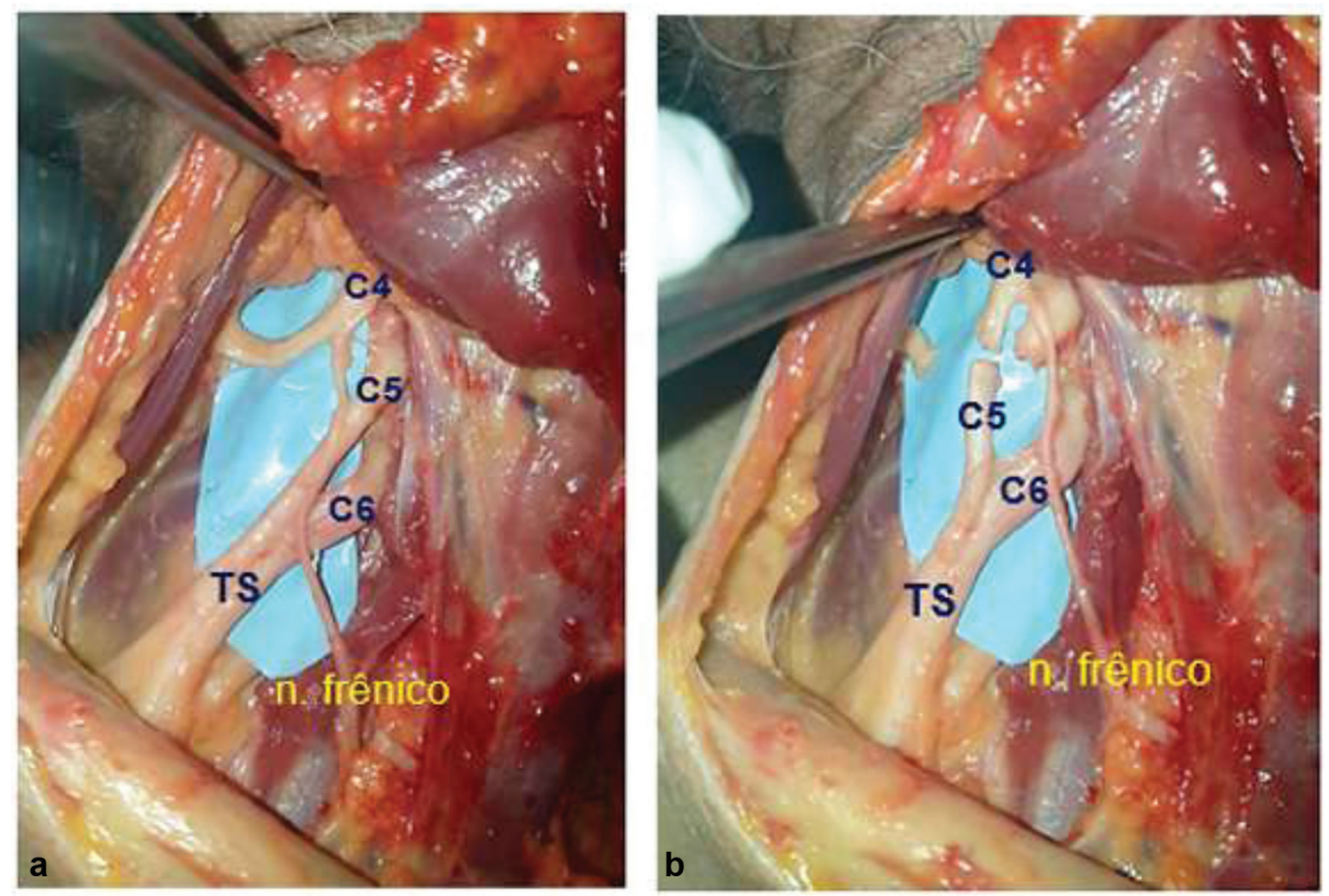

Fig. 2 Dissecção do plexo braquial direito. Demonstração das raízes de C4, C5, C6, tronco superior (TS) e origem do nervo frênico (a). Secção de C4 distal a emergência do nervo frênico e simulação da neurotização com a raiz de C5 que também foi seccionada(b). 
Tabela 1 Dados antropométricos

\begin{tabular}{|l|l|l|l|l|l|}
\hline $\mathbf{N}^{\circ}$ & Idade & Lado & Sexo & $\begin{array}{l}\text { Altura } \\
(\mathbf{c m})\end{array}$ & $\begin{array}{l}\text { Peso } \\
(\mathrm{Kg})\end{array}$ \\
\hline $\mathbf{1}$ & 52 & $\mathrm{D}$ & $\mathrm{M}$ & 167 & 61 \\
\hline $\mathbf{2}$ & 42 & $\mathrm{D}$ & $\mathrm{M}$ & 182 & 76 \\
\hline $\mathbf{3}$ & 66 & $\mathrm{E}$ & $\mathrm{M}$ & 175 & 74 \\
\hline $\mathbf{4}$ & 58 & $\mathrm{D}$ & $\mathrm{M}$ & 175 & 86 \\
\hline $\mathbf{5}$ & 72 & $\mathrm{E}$ & $\mathrm{F}$ & 164 & 68 \\
\hline $\mathbf{6}$ & 86 & $\mathrm{D}$ & $\mathrm{F}$ & 163 & 55 \\
\hline $\mathbf{7}$ & 86 & $\mathrm{E}$ & $\mathrm{F}$ & 163 & 55 \\
\hline $\mathbf{8}$ & 85 & $\mathrm{D}$ & $\mathrm{F}$ & 157 & 42 \\
\hline $\mathbf{9}$ & 85 & $\mathrm{E}$ & $\mathrm{F}$ & 157 & 42 \\
\hline 10 & 38 & $\mathrm{D}$ & $\mathrm{F}$ & 160 & 62 \\
\hline 11 & 38 & $\mathrm{E}$ & $\mathrm{F}$ & 160 & 62 \\
\hline 12 & 47 & $\mathrm{D}$ & $\mathrm{M}$ & 176 & 78 \\
\hline 13 & 47 & $\mathrm{E}$ & $\mathrm{M}$ & 176 & 78 \\
\hline 14 & 62 & $\mathrm{D}$ & $\mathrm{M}$ & 168 & 70 \\
\hline 15 & 62 & $\mathrm{E}$ & $\mathrm{M}$ & 168 & 70 \\
\hline média & 61,7 & & & 167,4 & 65,3 \\
\hline máximo & 86 & & & 182 & 86 \\
\hline mínimo & 38 & & & 157 & 42 \\
\hline
\end{tabular}

\section{Discussão}

São conhecidas as características anatômicas dos plexos cervical e braquial na região cervical, assim como a distribuição dos seus diferentes ramos no pescoço e no membro superior. ${ }^{18}$ No caso do plexo cervical, a raiz de $\mathrm{C} 4$ tem uma ampla distribuição na região cervical, e a sua porção sensitiva inerva a pele da região supraclavicular através dos nervos supraclaviculares (com contribuição também de C3). Já sua porção motora contribui na inervação de diferentes músculos, principalmente cervicais e apendiculares, sendo que, o seu ramo mais importante é o nervo frênico, que é formado por contribuições de $\mathrm{C} 3, \mathrm{C} 4$ e C5, responsável pela inervação motora do músculo diafragma. A raiz de C4 também fornece ramos para os músculos pré-vertebrais (longo do pescoço e longo da cabeça), ramos para os músculos escalenos e elevador da escápula, assim como ramos comunicantes para o nervo acessório que inerva o músculo trapézio. ${ }^{19}$ Os músculos mencionados não recebem inervação exclusiva de C4: as raízes adjacentes ao plexo cervical também contribuem com a sua inervação. Devido a essa inervação múltipla, a secção da raiz de C4 não provocaria déficit neurológico, ou ele seria mínimo. Uma exceção a isto é o ramo para o nervo frênico, uma vez que C4 contribui com a maior quantidade de fibras motoras em relação a C3 e C5 na sua formação. Na técnica que propomos, este ramo é preservado.

Tabela 2 Características morfológicas das raízes nervosas

\begin{tabular}{|c|c|c|c|c|c|c|c|}
\hline $\mathrm{N}^{\circ}$ & $\begin{array}{l}\text { C4, } \\
\text { comprimento } \\
\text { após origem } \\
\text { do nervo } \\
\text { frênico mm }\end{array}$ & $\begin{array}{l}\text { C5, } \\
\text { comprimento } \\
\text { após origem } \\
\text { do nervo } \\
\text { frênico mm }\end{array}$ & $\begin{array}{l}\text { C5, } \\
\text { comprimento } \\
\text { antes da origem } \\
\text { do nervo } \\
\text { frênico } \mathrm{mm}\end{array}$ & $\begin{array}{l}\mathrm{C} 5 \text {, } \\
\text { comprimento } \\
\text { total }\end{array}$ & $\begin{array}{l}\mathrm{C} 6 \text {, } \\
\text { comprimento } \\
\mathrm{mm}\end{array}$ & $\begin{array}{l}\text { Espaço } \\
\text { (Intervalo) } \\
\text { entre C4 e } \\
\text { C5 mm }\end{array}$ & $\begin{array}{l}\text { Diferencia } \\
\text { entre o } \\
\text { comprimento } \\
\text { de C4 e C5 }\end{array}$ \\
\hline 1 & 13 & 19 & 10 & 29 & 18 & 10 & 16 \\
\hline 2 & 14 & 18 & 8 & 26 & 19 & 10 & 12 \\
\hline 3 & 13 & 20 & 7 & 27 & 18 & 10 & 14 \\
\hline 4 & 11 & 20 & 7 & 27 & 20 & 11 & 16 \\
\hline 5 & 11 & 23 & 5 & 28 & 15 & 9 & 17 \\
\hline 6 & 13 & 18 & 7 & 25 & 16 & 9 & 12 \\
\hline 7 & 12 & 18 & 6 & 24 & 15 & 9 & 12 \\
\hline 8 & 13 & 21 & 7 & 28 & 17 & 9 & 15 \\
\hline 9 & 12 & 21 & 8 & 29 & 18 & 9 & 17 \\
\hline 10 & 12 & 19 & 8 & 27 & 18 & 10 & 15 \\
\hline 11 & 11 & 19 & 7 & 26 & 17 & 10 & 15 \\
\hline 12 & 14 & 20 & 8 & 28 & 18 & 11 & 14 \\
\hline 13 & 15 & 20 & 8 & 28 & 19 & 11 & 13 \\
\hline 14 & 12 & 18 & 9 & 27 & 17 & 10 & 15 \\
\hline 15 & 13 & 18 & 8 & 26 & 16 & 10 & 13 \\
\hline Média & 12,6 & 19,5 & 7,5 & 27,0 & 17,4 & 9,9 & 14,4 \\
\hline Máximo & 15 & 23 & 10 & 33 & 20 & 11 & 17 \\
\hline Mínimo & 11 & 18 & 5 & 23 & 15 & 9 & 12 \\
\hline
\end{tabular}


No plexo braquial, as fibras provenientes de C5 estão destinadas à inervação do ombro, e a sua lesão provoca déficit sensitivo na região lateral do mesmo e déficit para rotação externa e abdução. Os ramos que se originam da porção proximal de C5 (dorsal da escápula e contribuições para os nervos frênico e torácico longo) são considerados comprometidos pela avulsão de C5 e, portanto, foram seccionados para facilitar a mobilização da raiz.

Atualmente, as neurotizações mais usadas para reativar os movimentos do ombro são a transferência do nervo acessório para o nervo supraescapular, com o objetivo de estabilizar o ombro e dar algum grau de rotação externa ao mesmo, e a transferência de um dos ramos do nervo radial que inerva o músculo do tríceps para o nervo axilar, reativando a contração do músculo deltoide, e devolvendo assim a abdução do ombro. Abdouni et al. ${ }^{12}$ demonstraram que neurotização isolada do acessório tende a apresentar resultados frustrantes para a função do ombro. Além disso, a transferência do nervo acessório impossibilita usá-lo no futuro, em uma eventual neurotização de um retalho muscular livre. Por outro lado, a neurotização do nervo axilar tem como pré-requisito a integridade do nervo radial, restringindo-se o procedimento às lesões mais altas (C5-C6) do plexo braquial.

Yamada et al. ${ }^{14-16}$ descreveram a transferência das raízes de C3 e C4 para reconstrução das avulsões do tronco superior (C5 e C6); porém, a técnica proposta necessitava a interposição de enxerto nervoso para conectar as raízes, aumentando o trajeto a ser percorrido pela regeneração nervosa, além da dupla sutura que o uso do enxerto implica. Tsai et al. ${ }^{6}$ conseguiram, num estudo anatômico, coaptar a porção avulsionada de $\mathrm{C} 5$ à raiz de $\mathrm{C} 4$ sem a necessidade de enxerto nervoso, mas para isto houve a necessidade de laminectomia e dissecção intracanal, o que tornaria o procedimento tecnicamente mais difícil e com maior morbidade.

Considerando que as raízes de C4 e C5 têm um trajeto mais ou menos paralelo entre si, o comprimento maior de $\mathrm{C} 5 \mathrm{em}$ relação a $\mathrm{C} 4$, associado à mobilização das raízes, permite cobrir o espaço entre ambas as raízes sem a necessidade de utilizar enxerto de nervo. Por exemplo, no $1^{\circ}$ caso, o espaço entre as raízes (ao nível dos forames vertebrais) que deveria ser coberto foi de $10 \mathrm{~mm}$ e a diferença entre o comprimento de C4 (após a emergência do $\mathrm{n}$. frênico) e o comprimento total de $\mathrm{C} 5$ foi de $16 \mathrm{~mm}$, ou seja, mais do que o necessário para cobrir o espaço e realizar neurotização sem tensão. Em todos os casos o comprimento total de $\mathrm{C} 5$ maior que $\mathrm{C} 4$, e mostrou ser suficiente para cobrir o espaço (intervalo) entre essas duas raízes e realizar a neurotização sem tensão. No presente estudo, mobilizamos C5 para chegar até C4, com as medições no cadáver realizadas a partir do foramen vertebral. Nas lesões por avulsão, é provável que comprimento disponível de C5 seja ainda maior, mesmo com a necessidade de ressecção do segmento proximal avulsionado.

Embora a neurotização de elementos sensitivos e motores do plexo cervical para reparação de lesões do plexo braquial tenham sido descritos há muito tempo, ${ }^{20}$ os ramos motores de C3 e C4 usualmente transferidos são muito finos e com poucas fibras nervosas, não proporcionando assim bons resultados nas publicações anteriores. O objetivo desta técnica foi incluir uma maior quantidade de fibras motoras na neurotização, levando, junto com a raiz de $\mathrm{C} 4$, as fibras motoras que estariam destinadas para os músculos escalenos, romboides, elevador da escápula e músculo trapézio (ramos comunicantes para o nervo espinhal acessório). ${ }^{18,21,22}$

Recentemente, operamos 3 pacientes com lesões superiores pré-ganglionares confirmados com ressonância magnética (RM), sendo 2 com lesão $\mathrm{C} 5-\mathrm{C} 6-\mathrm{C} 7$, em que não havia a possibilidade da transferência do ramo do nervo radial para o nervo axilar e 1 paciente com lesão C5-C6. Além da neurotização de C4 para C5 (-Figura 3), realizamos também a cirurgia de Oberlin para reinervação do bíceps braquial. Em todos os casos foi possível a sutura direta, sem enxerto de nervo, e não foi observado, no pós-operatório, nenhum déficit
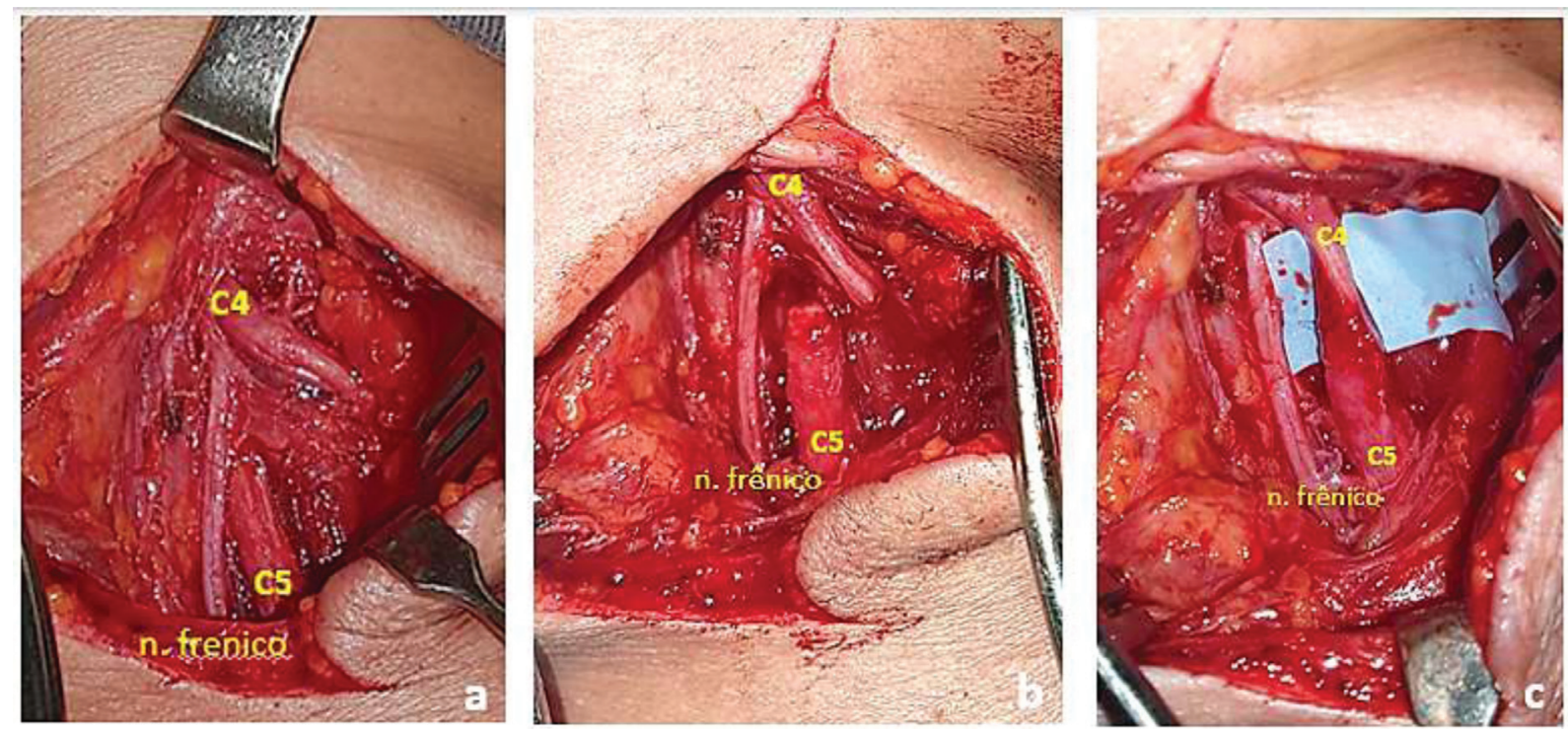

Fig. 3 Imagens intraoperatórias da região cervical esquerda. Disseção mostrando as raízes de C4 e C5 além do nervo frênico (a). Raiz de C4 seccionada distal a origem do nervo frênico e raiz de C5 seccionada logo após da sua saída do forame intervertebral (b). Neurorrafia entre C4 e C5 com preservação do nervo frênico (c). 
relacionado ao sacrifício da raiz de C4. Os resultados, embora preliminares, uma vez que o tempo de evolução pós-operatória variou entre 4 e 6 meses, são animadores. Nosso intuito é realizar uma série de casos maior e aguardar os desfechos clínicos para divulgar os resultados posteriormente.

A técnica apresentada permitirá a reinervação da musculatura do ombro, devolvendo tanto a rotação externa como a abdução. Tudo isso é realizado em uma única neurotização, reduzindo assim o tempo cirúrgico e a morbidade no pósoperatório. O nervo acessório é poupado e poderá ser usado, caso necessário, para outra neurotização, como na transferência livre funcional do musculo grácil. Além disso, essa técnica poderá auxiliar no tratamento das complexas lesões totais do plexo braquial.

\section{Conclusões}

Em todas as dissecções foi possível simular a transferência de C4 para C5, realizando sutura direta, sem tensão. A técnica mostra-se segura, porém requer uma equipe capacitada e um cirurgião com experiência e conhecimento amplo da anatomia da região abordada.

\section{Suporte Financeiro}

Não houve suporte financeiro de fontes públicas, comerciais, ou sem fins lucrativos.

\section{Conflito de Interesses}

Os autores declaram não haver conflito de interesses.

\section{Referências}

1 Flores LP. Estudo epidemiológico das lesões traumáticas de plexo braquial em adultos. Arq Neuropsiquiatr 2006;64(01):88-94

2 Mello Júnior JS, Souza TCR, Andrade FG, et al. Perfil Epidemiológico de Pacientes com Lesão Traumática do Plexo Braquial avaliados em um Hospital Universitário no Rio de Janeiro. Rev Bras Neurol 2012;48(03):5-8

3 Rezende MR, Rabelo NTA, Silveira Júnior CC, Petersen PA, De Paula EJL, Mattar Júnior R. Resultado na neurotização do nervo ulnar para o músculo bíceps braquial na lesão do plexo braquial. Acta Ortop Bras 2012;20(06):317-323

4 Sedain G, Sharma MS, Sharma BS, Mahapatra AK. Outcome after delayed Oberlin transfer in brachial plexus injury. Neurosurgery 2011;69(04):822-827, discussion 827-828

5 Venkatramani H, Bhardwaj P, Faruquee SR, Sabapathy SR. Functional outcome of nerve transfer for restoration of shoulder and elbow function in upper brachial plexus injury. J Brachial Plex Peripher Nerve Inj 2008;3:15

6 Tsai YJ, Su FC, Hsiao CK, Tu YK. Comparison of objective muscle strength in C5-C6 and C5-C7 brachial plexus injury patients after double nerve transfer. Microsurgery 2015;35(02):107-114
7 Kaiser R, Waldauf P, Ullas G, Krajcová A Epidemiology, etiology, and types of severe adult brachial plexus injuries requiring surgical repair: systematic review and meta-analysis. Neurosurg Rev 2020;43(02):443-452

8 Ali ZS, Heuer GG, Faught RW, et al. Upper brachial plexus injury in adults: comparative effectiveness of different repair techniques. J Neurosurg 2015;122(01):195-201

9 Verdins K, Kapickis M. Oberlin's Transfer: Long Term Outcomes. J Hand Surg Asian Pac Vol 2018;23(02):176-180

10 Bertelli JA, Ghizoni MF. Reconstruction of C5 and C6 brachial plexus avulsion injury by multiple nerve transfers: spinal accessory to suprascapular, ulnar fascicles to biceps branch, and triceps long or lateral head branch to axillary nerve. J Hand Surg Am 2004;29(01):131-139

11 Liverneaux PA, Diaz LC, Beaulieu JY, Durand S, Oberlin C. Preliminary results of double nerve transfer to restore elbow flexion in upper type brachial plexus palsies. Plast Reconstr Surg 2006;117 (03):915-919

12 Abdouni YA, Checoli GF, Salles HC, da Costa AC, Chakkour I, Fucs PMMB. Assessment of the results of accessory to suprascapular nerve transfer. Acta Ortop Bras 2018;26 (05):332-334

13 Malessy MJ, de Ruiter GC, de Boer KS, Thomeer RT. Evaluation of suprascapular nerve neurotization after nerve graft or transfer in the treatment of brachial plexus traction lesions. J Neurosurg 2004;101(03):377-389

14 Yamada S, Peterson GW, Soloniuk DS, Will AD. Coaptation of the anterior rami of $\mathrm{C}-3$ and $\mathrm{C}-4$ to the upper trunk of the brachial plexus for cervical nerve root avulsion. J Neurosurg 1991;74(02): 171-177

15 Yamada S, Lonser RR, Iacono RP, Morenski JD, Bailey L. Bypass coaptation procedures for cervical nerve root avulsion. Neurosurgery 1996;38(06):1145-1151, discussion 1151-1152

16 Yamada S, Lonser RR, Colohan AR, Yamada SM, Won DJ. Bypass coaptation for cervical root avulsion: indications for optimal outcome. Neurosurgery 2009;65(4, Suppl):A203-A211

17 Yang KX, Zhang SH, Ge DW, Sui T, Chen HT, Cao XJ. A novel extradural nerve transfer technique by coaptation of C4 to C5 and C7 to 6 for treating isolated upper trunk avulsion of the brachial plexus. J Biomed Res 2018;32(04):298-304

18 Netter FH. Atlas of human anatomy. 6th ed. Philadelphia: Elsevier; 2014

19 Ferrao A, Megali R, Papais RM. Surgical anatomy of the spinal accessory nerve: how to avoid injuries to the posterior cervical triangle during surgical procedures. Rev Bras Cir Plást 2009;24 (04):400-413

20 Brunelli G. Neurotization of avulsed roots of the brachial plexus by means of anterior nerves of the cervical plexus. Int J Microsurg 1980;55:529-531

21 Tubbs RS, Shoja Mohamm Adali M, Loukas M, et al. Study of the cervical plexus innervation of the trapezius muscle. J Neurosurg Spine 2011;14:626-629

22 Gavid M, Mayaud A, Timochenko A, Asanau A, Prades JM. Topographical and functional anatomy of trapezius muscle innervation by spinal accessory nerve and C2 to C4 nerves of cervical plexus. Surg Radiol Anat 2016;38(08):917-922 